\begin{tabular}{|c|c|}
\hline Title & One-Pot Room-Temperature Synthesis of Mg Containing MCM- 41 Mesoporous Silica for A Idol Reactions \\
\hline Author(s) & Fujita, Shin-Ichiro; Segawa, Shinji; Kawashima, Kazuki; Nie, X uejing; Erata, Tomoki; A rai, Masahiko \\
\hline Citation & $\begin{array}{l}\text { Journal of materials science \& technology, 34(12), 2521-2528 } \\
\text { https://doi.org/10.1016/.jmst.2016.08.025 }\end{array}$ \\
\hline Issue Date & 201812 \\
\hline Doc URL & http:/hdl.handle.net/2115/79189 \\
\hline Rights & 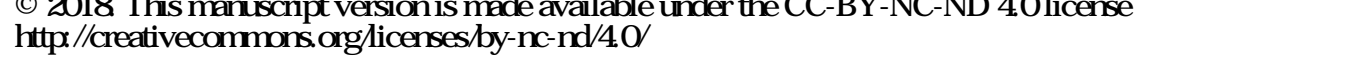 \\
\hline Rights(URL) & http://creativecommons.org/icenses/by-nc-nd/4.0/ \\
\hline Type & article (author version) \\
\hline File Information & 2016-0916R1.pdf \\
\hline
\end{tabular}

Instructions for use 


\section{One-pot Room-Temperature Synthesis of Mg Containing MCM-41 Mesoporous Silicas for Aldol Reactions}

Shin-ichiro Fujita*, Shinji Segawa, Kazuki Kawashima, Xuejing Nie, Tomoki Erata, Masahiko Arai

Division of Applied Chemistry, Faculty of Engineering, Hokkaido University, Sapporo 060-8628, Japan

* Corresponding author. Ph.D.; Tel.: +81 117066596; Fax: +86 117067556.

E-mail address: sfuji@enghokudai.ac.jp (S. Fujita).

One-pot synthesis of Mg containing MCM-41 (Mg-MCM-41) materials was carried out by a room temperature (RT) method and by a hydrothermal (HT) method and they were used for aldol condensation of 4-nitrobenzaldehyde and acetone and self-condensation of acetone. The RT method can prepare MCM-41 materials containing large amounts of Mg while maintaining the structural characteristics of MCM-41 even at very low Si/Mg ratios (large Mg loadings), but the HT method cannot. The RT method can also give more active catalysts than the HT method, because the catalysts prepared by the former method are more basic than those prepared by the latter one. The characterization results indicate that Mg atoms in Mg-MCM-41 prepared by the RT method exist as MgO dispersing well on the wall surface of pores, while those in Mg-MCM-41 prepared by the HT method are included in the bulk with a smectite-like structure.

Key words: Aldol condensation, Solid base catalyst, Mg incorporated mesoporous silica, magic angle spinning nuclear magnetic resonance

\section{Introduction}

MCM-41 discovered by the Mobil scientists ${ }^{[1,2]}$ is the most famous member of mesoporous silicious materials. It possesses a hexagonally ordered array of uniform pores, large surface areas, and large pore volumes. The morphology of MCM-41 strongly depends on 
the preparation variables such as template type, silica source, relative concentration, $\mathrm{pH}$ value, aging conditions and so on ${ }^{[3]}$. Conventionally, MCM-41 is synthesized by hydrothermal treatment of a solution containing the silica source and the template at elevated temperatures around $100-150{ }^{\circ} \mathrm{C}$ for a few days. On the other hand, the room-temperature synthesis of silicious MCM-41 is also possible ${ }^{[4-7]}$. It is a rapid method under mild conditions consuming less energy and time than the hydrothermal synthesis.

The diffusion of large molecules inside the mesopores of MCM-41 would be faster compared with widely used microporous zeolites. Such textural properties of MCM-41 can be advantageous for its application in catalysis. Despite of this advantage, silicious MCM-41 itself is usually catalytically low active or inactive because its chemical nature is very similar to that of amorphous silica and hence it requires functionalization to be used as catalysts. To generate catalytically active sites, the incorporation of such heteroatoms as $\mathrm{Al}^{[8-16]}, \mathrm{V}^{[17]}, \mathrm{Ti}^{[18-21]}, \mathrm{Mn}$ ${ }^{[22,23]}, \mathrm{Fe}^{[24]}$, and $\mathrm{B}^{[25]}$ was examined. Among the MCM-41 materials containing heteroatoms, Al containing ones (Al-MCM-41) have been most extensively studied because of their importance as solid acid catalysts ${ }^{[15]}$. The incorporation of Al into silica framework can produce surface silanol groups with enhanced acidic strength and the amount of those acidic sites can be increased with increasing the amount of $\mathrm{Al}$ incorporated; however, the incorporation of even small amounts of $\mathrm{Al}$ into MCM-41 could cause the decreases in the hexagonally ordered structure, the surface area, and the pore volume ${ }^{[12-14]}$. Such negative effects of $\mathrm{Al}$ incorporation on the textural properties of Al-MCM-41 can be avoided by some degree with the room-temperature synthesis ${ }^{[26-29]}$.

Development of solid base catalysts is still an important issue from both the academic and industrial viewpoints ${ }^{[30-34]}$. There are several ways to prepare solid base catalysts from MCM-41 ${ }^{[35-37]}$. One of them is the functionalization of MCM-41 with basic metal oxides like $\mathrm{MgO}$ and $\mathrm{CaO}{ }^{[38-40]}$. Yu et al. ${ }^{[38]}$ reported that, when $20 \mathrm{wt} \%-\mathrm{MgO}$ was supported on Al-MCM-41 by impregnation of Mg acetate followed by calcination, the decreases in the hexagonally ordered structure, the surface area, and the pore volume were also observed. Such a decrease in the hexagonally ordered structure did not occur when $10 \mathrm{wt} \%$-MgO was supported on pure silica MCM-41; however, the surface area and the pore volume were decreased probably due to the blockade of mesopores ${ }^{[39]}$. Thus, even with the impregnation method, supporting a large amount of $\mathrm{MgO}$ could also show a negative effect on the textural 
properties of MCM-41.

Under these circumstances, our group initiated the one-pot room temperature synthesis of Mg containing MCM-41 (Mg-MCM-41). In the present study, two series of Mg-MCM-41 materials were prepared by a room temperature (RT) method and by a hydrothermal (HT) method, and differences in their structural and catalytic properties were investigated. It has been shown that the synthesis of Mg-MCM-41 materials having the characteristic ordered structure of MCM-41 is possible by the RT method in one-pot even at small Si/Mg ratios (i.e. large Mg loadings), while the preparation of such Mg-MCM-41 is difficult by the HT synthesis. Using those Mg-MCM-41 materials, aldol condensation of 4-nitrobenzaldehyde and acetone and self-condensation of acetone (Scheme 1) were carried out as model base-catalyzed reactions. It was found that the RT method can give more active catalysts than the hydrothermal synthesis. The difference in the catalytic activity between the two synthesis methods was discussed on the basis of the characterization results obtained by several techniques including X-ray fluorescence, XRD, TEM, $\mathrm{N}_{2}$ adsorption, measurement of base strength, ${ }^{29}$ Si NMR, and UV/Vis spectroscopy.

\section{Experimental}

\subsection{Catalyst preparation}

Two series of MCM-41 materials were prepared by a room temperature (RT) method and by a hydrothermal (HT) method. The RT method was similar to that in the literature ${ }^{[29]}$. For the preparation of pure silica MCM-41, $5 \mathrm{~cm}^{3}$ of 28\% ammonia aqueous solution, $1.67 \mathrm{~g}$ of hexadecyltrimethylammonium-bromide (CTAB), and $5 \mathrm{~cm}^{3}$ tetraethylorthosilicate (TEOS) were successively added to $500 \mathrm{~cm}^{3}$ of distilled water under stirring. Then, the mixture was further stirred at room temperature for $1 \mathrm{~h}$. The resulting precipitates were collected by filtration and washed with distilled water until the $\mathrm{pH}$ value of water decreased to 7 . The solid materials obtained were dried in an oven at $333 \mathrm{~K}$ for $12 \mathrm{~h}$ and calcined in air at $573 \mathrm{~K}$ for $2 \mathrm{~h}$ and then at $873 \mathrm{~K}$ for $6 \mathrm{~h}$. For the calcination, the heating rate was $2 \mathrm{~K} \mathrm{~min}^{-1}$. The sample obtained is designated as MCM-RT. MCM-41 samples incorporated with $\mathrm{Mg}$ and co-incorporated with $\mathrm{Mg}$ and $\mathrm{Al}$ were also prepared via the RT method. For the preparation, required amounts of $\mathrm{Mg}$ ethoxide and $\mathrm{Al}$ chloride hexahydrate were further added to the 
above-mentioned ammonia, CTAB, and TEOS solution. Following procedures were the same as used in the preparation of MCM-RT. The Si/Mg ratio was changed in a range from 1.5 to 10 (1.5, 2.7, 5, and 10), while the $\mathrm{Si} / \mathrm{Al}$ ratio was set at 30 . These samples are designated as Mg-MCM-RT and Mg-Al-MCM-RT.

For comparison, corresponding MCM-41 samples were prepared with a HT method (designated as MCM-HT, Mg-MCM-HT, and Mg-Al-MCM-HT) similar to that in the literature [16]. For the preparation of these samples, to a mixture of $66.8 \mathrm{~cm}^{3}$ of distilled water and 4.7 $\mathrm{cm}^{3}$ of $28 \%$ ammonia aqueous solution, $1.4 \mathrm{~g}$ of CTAB was dissolved, and the solution was stirred at room temperature for $30 \mathrm{~min}$. Then, $5.36 \mathrm{~g}$ of TEOS was added dropwise under stirring over a period of 15 min. For the preparation of Mg-MCM-HT and Mg-Al-MCM-HT, required amounts of the $\mathrm{Mg}$ and $\mathrm{Al}$ precursors were further added after the addition of TEOS. The resulting mixture was kept at room temperature for $12 \mathrm{~h}$ under stirring. The mixture was moved to a $100 \mathrm{~cm}^{3}$ Teflon-lined autoclave and heated in an oven at $383 \mathrm{~K}$ for $72 \mathrm{~h}$ without stirring. The procedures following the hydrothermal treatment were the same as employed in the RT synthesis method.

Among the Mg containing MCM-41 samples, the characterization was mostly carried out for the ones of a Si/Mg ratio of 2.7, corresponding to the $\mathrm{MgO}$ loading of $20 \mathrm{wt} \%$.

\subsection{Catalyst characterization}

The catalyst compositions were determined by X-ray fluorescence using a JEOL JSX-3100RII spectrometer and they were found to be almost the same with the nominal ones. X-ray diffraction (XRD) was used to examine the structure of samples. The small- and large-angle XRD patterns were recorded on a RIGAKU RINT 2200 ULTIMA diffractometer using $\mathrm{Ni}$ filtered $\mathrm{Cu} K_{\alpha}$ radiation. Transmission electron microscopy (TEM) images were collected on a JEOL JEM-2000FX microscopy. BET surface area, total pore volume, and pore size distribution were obtained from $\mathrm{N}_{2}$ adsorption-desorption isotherms using a gas sorption analyzer (YUASA IONICS, NOVA 1000). Before analysis, all the samples were dried at $423 \mathrm{~K}$ for $2 \mathrm{~h}$ under vacuum. The pore size distribution was evaluated by the Barret-Joyner-Halenda method. 
The base strength of the samples were examined by using several Hammet indicators of 4-nitrophenol $(\mathrm{pKa}=7.1)$, cresol red $(\mathrm{pKa}=8.0)$, Thymol blue $(\mathrm{pKa})=8.9$, phenolphthalein $(\mathrm{pKa}=9.3)$, and 4-nitroanilline $(\mathrm{pKa}=18.4)$. A certain amount of the sample was dispersed in an ethanol solution of the indicator for a few minutes. Then, it was checked by naked eyes whether the sample turned to the deprotonated color of the indicator. By changing the indicator employed, the base strength of the samples was determined.

The chemical environments of Si atoms were analyzed by ${ }^{29} \mathrm{Si}$ MAS (Magic Angle Spinning) NMR spectroscopy with and without CP (cross-polarization). All NMR experiments were performed on a Bruker MSL 400 spectrometer (for details, see Supporting Information). Diffuse reflectance UV-vis spectra were also measured on a Shimadzu UV-3100PC spectrometer to examine the state of $\mathrm{Mg}$ atoms.

\subsection{Catalytic reactions}

All reaction runs were carried out in a $100 \mathrm{~cm}^{3}$ Teflon-lined autoclave. For the aldol condensation of 4-nitrobenzaldehyde and acetone, the reactor was charged with $10 \mathrm{~cm}^{3}$ of acetone, $0.5 \mathrm{mmol}$ of 4-nitrobenzene, and $50 \mathrm{mg}$ of catalyst and purged with $\mathrm{N}_{2}$ several times. Then, the reaction was conducted at $353 \mathrm{~K}$ for $7 \mathrm{~h}$ under stirring. After the reaction, the mixture was cooled, separated from the solid catalyst, and analyzed by a gas chromatograph with a flame ionization detector. The self-condensation of acetone was carried out in the same reactor with $10 \mathrm{~cm}^{3}$ of acetone and $50 \mathrm{mg}$ of the catalyst at $353 \mathrm{~K}$ for $2 \mathrm{~h}$. The procedures for this reaction were the same as for the aldol condensation mentioned above.

\section{Results and Discussion}

\subsection{Textural properties}

Fig. 1 represents the pore size distribution curves of several samples. It shows that all the samples prepared by the RT method contained uniform mesopores around $2.4 \mathrm{~nm}$, which is one of the structural characteristics of MCM-41, although the curves of Mg-MCM-RT and Mg-Al-MCM-RT were slightly broader (Fig. 1a). It was also found that there were not large differences in the surface area among these samples prepared by the RT method (Table 1). The 
surface areas of Mg-MCM-RT and Mg-Al-MCM-RT were larger than 80\% of that of MCM-RT. In contrast to the RT method, the pore size distribution curves of the samples prepared by the HT method were distinctly changed by the incorporation of Mg. Compared to MCM-HT, the intensities of the peaks at $2.4 \mathrm{~nm}$ in the distribution curves of Mg-MCM-HT and Mg-Al-MCM-HT were much weaker (Fig. 1b), revealing the collapse of the uniform mesopore structure by the incorporation of Mg. In addition, the surface areas of Mg-MCM-HT and Mg-Al-MCM-HT were also much lower than that of MCM-HT (Table 1). In the pore size distribution curve of MCM-HT, the peak at $2.4 \mathrm{~nm}$ was accompanied by the one at $4 \mathrm{~nm}$. The latter peak was absent in the distribution curve of MCM-RT and this would probably result from the coalescence of two adjacent mesopores of $2.4 \mathrm{~nm}$ in the diameter. Thus, for the preparation of MCM-41 material containing Mg, it would be better to employ the RT method than the HT method from the viewpoint of the pore structure.

Fig. 2 illustrates the small-angle XRD patterns of several samples. In the XRD pattern of MCM-RT, the typical peaks attributed to the hexagonally ordered structure of mesopore array were observed at $2.3^{\circ}, 3.9^{\circ}$, and $4.5^{\circ}$, which were indexed [100], [110], and [200], respectively [1,28,37]. For Mg-MCM-RT and Mg-Al-MCM-RT, the [100] reflection became broader and the [110] and [200] reflections were not discernible. Those would result from the short-range order of the mesopore arrays in these samples; however, one can say that the hexagonally ordered structure would be preserved in them. For the corresponding samples prepared by the HT method, the [100] reflection was very weak (Mg-MCM-HT) or absent (Mg-Al-MCM-HT). In conformity with the results of the small-angle XRD measurements, the hexagonally ordered array could be observed in the TEM image of Mg-Al-MCM-RT, but not in that of Mg-Al-MCM-HT (Fig. 3). Thus, the order of mesopore array in the Mg containing sample also depended on the preparation method used.

As already described, the hexagonally ordered array of uniform mesopores, large surface areas, and large pore volumes are structural characteristics of MCM-41 materials. The above-mentioned results of $\mathrm{N}_{2}$ adsorption and XRD measurements reveal that the RT method can prepare MCM-41 materials containing large amounts of Mg while maintaining those structural characteristics, but this is very difficult by the HT method. The Si/Mg ratio of the Mg-MCM-RT sample in Table 1 and Figs. 1 and 2 was 2.7. It should be noted that this ratio corresponds to the Mg loading of $20 \mathrm{wt} \%$ in MgO. Even by the impregnation method, loading 
such a large amount of Mg was difficult because of the collapse of the hexagonally ordered structure and/or the blockage of mesopores ${ }^{[37,38]}$. To examine the further extension of the RT method, it was applied to prepare an Mg-MCM-RT sample of a smaller Si/Mg ratio of 1.5, which corresponds to $40 \mathrm{wt} \%$ in MgO. Even at this low ratio, the sample still had a large surface area of $865 \mathrm{~m}^{2} / \mathrm{g}$ and showed the characteristic peaks in its pore size distribution curve and small-angle XRD pattern (Fig. S1), although the peak intensities were slightly weaker compared to the sample of the $\mathrm{Si} / \mathrm{Mg}$ ratio of 2.7 . Thus, the RT method is excellent for the preparation of Mg incorporated MCM-41.

\subsection{Catalytic Performance}

As the model reaction, the aldol condensation of 4-nitrobenzaldehyde and acetone producing 4-hydroxy-4-(4-nitrophenyl)-butan-2-one and 4-(4-nitrophenyl)-3-buten-2-one was carried out using the catalysts containing various amounts of Mg. For this reaction, MCM-RT and MCM-HT were inactive and the catalytic activity was emerged by incorporating Mg. In Fig. 4, the conversion of 4-nitrobenzaldehyde is plotted against the amount of Mg incorporated. It is shown that both the catalytic activities of Mg-MCM-RT and Mg-Al-MCM-RT were enhanced with increasing the amount of Mg incorporated, while the activities of Mg-MCM-HT and Mg-Al-MCM-HT were not changed so much by increasing the Mg incorporation. Consequently, the catalysts prepared by the HT method exhibited much lower activity than those prepared by the RT method at high Mg loadings.

In Fig. 4, it is also seen that there is practically no difference in the activity between Mg-MCM-RT and Mg-Al-MCM-RT and the activity of Mg-Al-MCM-HT is lower than that of Mg-MCM-HT. These findings reveal that the Al incorporation into MCM-RT and -HT has no or negative effect on the catalytic activity. In the literature ${ }^{[41-44]}$, it has been reported that synergistic effect can be observed for the aldol condensation reaction over acid-base bifunctional solid catalysts. Such synergistic effect was absent in the present catalyst system.

As shown in Fig. 1, the mesopore volumes of Mg-MCM-HT and Mg-Al-MCM-HT were much smaller than those of Mg-MCM-RT and Mg-Al-MCM-RT. Over Mg-MCM-HT and Mg-Al-MCM-HT, therefore, the reaction would mostly proceed inside their micropores, which might limit the reaction by the diffusion resistance of the relatively large molecule of 
4-nitrobenzaldehyde. To examine this possibility, the self-condensation of acetone was carried out, because this reaction was considered to be less affected by the diffusion in the micropores. As shown in Fig. 5, Mg-MCM-RT was again found to be much more active than Mg-MCM-HT. So, the difference in the activity was caused by other reasons than the pore structure.

\subsection{Chemical properties}

To elucidate the reason for the difference in the catalytic activity by the preparation method, further characterization of the catalysts were conducted. The base strength of the catalysts was determined by using several $\mathrm{pH}$ indicators. Mg-MCM-RT ( $\mathrm{Si} / \mathrm{Mg}=2.7$ ) was colored by phenolphthalein $(\mathrm{pKa}=9.3)$ to its deprotonated color. In contrast, Mg-MCM-HT $(\mathrm{Si} / \mathrm{Mg}=2.7)$ was not colored by either phenolphthalein or cresol red $(\mathrm{pKa}=8.0)$, but colored by 4-nitrophenol (pKa = 7.1). With the other Mg-MCM-RT and Mg-MCM-HT samples of different $\mathrm{Si} / \mathrm{Mg}$ ratios, the same results were obtained, revealing that the base strength did not depend on the Si/Mg ratio. Thus, the basicity of Mg-MCM-RT is stronger than Mg-MCM-HT, resulting in the higher activity of Mg-MCM-RT as observed.

The quantification of those basic sites was examined by an acid-base titration. Unfortunately, however, it was unable because Mg species in the samples were easily dissolved into the acid solution.

Fig. 6 represents the large-angle XRD patterns of Mg-MCM-RT and of Mg-MCM-HT at a few different Si/Mg ratios. In the XRD patterns of Mg-MCM-RT at Si/Mg ratios of 2.7 and 1.5, two peaks assigned to $\mathrm{MgO}$ were observed at $42^{\circ}$ and $62^{\circ}$ along with a broad one of amorphous silica around $24^{\circ}$. In the XRD patterns of Mg-MCM-HT at the same Si/Mg ratios, the peaks of $\mathrm{MgO}$ could not be seen. Instead, three weak peaks were observed at $20^{\circ}, 35^{\circ}$, and $60^{\circ}$. Those peaks were ascribable to $\mathrm{Mg}$ containing smectite (Mg-smectite) ${ }^{[45]}$. Thus, the chemical states of $\mathrm{Mg}$ species are different between Mg-MCM-RT and Mg-MCM-HT at least at low Si/Mg ratios (high Mg loadings). Probably, the MgO-like and Mg-smectite-like species could exist in Mg-MCM-RT and Mg-MCM-HT, respectively, even at higher Si/Mg ratios (lower $\mathrm{Mg}$ loadings), although they could not be identified by XRD.

Diffuse reflectance UV-vis spectra of Mg-MCM-RT and of Mg-MCM-HT are represented 
in Fig. 7. Both catalysts showed the absorption bands at 250 and $273 \mathrm{~nm}$, which could be assigned to the absorption bands of Mg-O-Si species ${ }^{[46-48]}$ and of Mg-O pairs involving 3-coordinated $\mathrm{O}^{2-}$ ions ${ }^{[48,49]}$, respectively. The former band was predominant in the spectrum of Mg-MCM-RT, while it was weaker than the latter one in the spectrum of Mg-MCM-HT.

The presence of Mg-O-Si species was also identified by ${ }^{29}$ Si MAS NMR. Fig. 8 illustrates ${ }^{29}$ Si MAS NMR spectra of Mg-MCM-RT and Mg-MCM-HT. In the spectrum of Mg-MCM-RT, two signals at -110 and $-100 \mathrm{ppm}$ and a broad signal around $-80 \mathrm{ppm}$ were observed. The signals at -110 and -100 ppm would arise from Si atoms with four siloxane bonds and from those with three siloxane bonds and a hydroxyl group, respectively ${ }^{[47,50]}$. The broad signal around $-80 \mathrm{ppm}$ could be assigned to $\mathrm{Si}$ atoms in Mg-O-Si groups ${ }^{[51-53]}$. The structure of $\mathrm{Mg}$ species corresponding to this signal will be discussed later. In the spectrum of Mg-MCM-HT, two additional strong signals around -98 and -92 were observed. These could be related to the formation of Mg-smectite like species whose formation was detected by the large-angle XRD measurements (Fig. 6), because these two peaks were absent in the spectrum of MCM-HT (Fig. S2) and several magnesium silicate minerals and silica supported MgO catalysts could show resonance peaks in a region between -78 and $-98 \mathrm{ppm}{ }^{[47,51-53]}$. Surface sensitive ${ }^{29} \mathrm{Si}$ CP MAS NMR spectra of Mg-MCM-RT and Mg-MCM-HT were also measured (Fig. 9). Comparison of Figs. 8 and 9 clearly showed that the intensity of the signal around $-80 \mathrm{ppm}$ was enhanced in the CP spectrum of Mg-MCM-RT. Such enhancement was not observed for any signal for Mg-MCM-HT. These results reveal that Mg species involved in Mg-MCM-RT are located on the surface of silica [50]. Reversely, the absence of the enhancement in the signal intensity for Mg-MCM-HT suggests that most Mg species exist in the bulk of the catalyst.

On the basis of the results of large-angle XRD, UV-vis spectroscopy, and MAS NMR spectroscopy, further discussion was made on the structure of $\mathrm{Mg}$ species involved in the catalysts. As shown in Fig. 6, the XRD patterns of Mg-MCM-RT showed the presence of MgO over the catalysts. Because the peak intensity of $\mathrm{MgO}$ was weak, $\mathrm{MgO}$ would probably be finely dispersed on the silica surface. On the other hand, the UV-vis and MAS NMR measurements for Mg-MCM-RT detected Mg-O-Si species and those species were found to locate on the silica surface. The NMR signal around $-80 \mathrm{ppm}$ was previously assigned to amorphous magnesium silicate of $\mathrm{Mg}_{2} \mathrm{SiO}_{4}$, which was proposed to be formed as an intermediate during the heat treatment of chrysotile ${ }^{[51]}$. Taking account of the XRD results, 
however, the formation of such compound for Mg-MCM-RT was unlikely. Similar NMR signals were reported in the literature for MgO catalysts supported on silica or MCM-41and for a milled mixture of $\mathrm{Mg}(\mathrm{OH})_{2}$ and silica ${ }^{[47,53]}$. Thus, one can conclude that $\mathrm{Mg}$ atoms involved in Mg-MCM-RT exist as MgO that is finely dispersed on the surface inside the pores (Scheme 2a). Those MgO species would strongly interact with the surface consisting of silica, resulting in the formation of Mg-O-Si bonds. For Mg-MCM-HT, the XRD measurement showed the formation of Mg-smectite like species (Fig. 6). Smectite is one of the layered clay minerals in which one layer consists of one octahedral sheet sandwiched by two tetrahedral sheets. The octahedral sheet contains divalent cations like $\mathrm{Mg}^{2+}$ surrounded by four oxygen atoms and the tetrahedral sheet contains $\mathrm{Si}^{4+}$ cations surrounded by six oxygen atoms. Mg-smectite can be synthesized from co-precipitates produced from $\mathrm{MgCl}_{2}$ and water-glass by hydrothermal treatments under certain conditions without using any template ${ }^{[45,54]}$. When an un-calcined Mg-MCM-RT sample was hydrothermally treated as for the HT method and then calcined, the resulting sample showed the three XRD peaks of Mg-smectite. So, it is highly probable that Mg-smectite like species are also formed in the course of the hydrothermal treatment for the HT method and an appreciable fraction of $\mathrm{Mg}$ atoms is included in the bulk with the smectite-like structure (Scheme 2b). Of course, the formation of MgO species dispersing on the pore surface, which are supposed to exist on Mg-MCM-RT, cannot completely be excluded for Mg-MCM-HT. But the amount of such MgO species over Mg-MCM-HT would be small, as suggested by the NMR results. Thus, the state of $\mathrm{Mg}$ atoms is different by the preparation method.

Angelici et al. [48] prepared silica supported MgO catalysts by either kneading or co-precipitation and found that the catalysts prepared by the former was stronger base than the ones prepared by the latter. They described that an intimate contact between the two components is significant for the basicity, but full mixing of them is not. The situation is similar for the present catalyst system. As mentioned above, the base strength of the catalyst was different by the preparation method. This can be explained by the difference in the chemical state of $\mathrm{Mg}$ atoms. In the Mg-smectite structure, the chemical nature of $\mathrm{Mg}$ atoms in the tetrahedral sheet would be different from that of bulk MgO because of the 2-dimentional structure. Furthermore, even if those Mg atoms have the catalytic activity as base, it would be hindered by the octahedral sheets sandwiching the tetrahedral sheet. Consequently, the basicity of Mg-smectite is weak as previously revealed by $\mathrm{CO}_{2}$ temperature programmed desorption experiments ${ }^{[54]}$. Therefore, Mg atoms in Mg-MCM-HT would show weak basicity. On the 
other hand, Mg atoms in Mg-MCM-RT exist as MgO dispersing on the wall surface of pores and hence its basicity would be similar to that of bulk MgO. Thus, Mg-MCM-RT is stronger base than Mg-MCM-HT.

As shown in Fig. 4, Mg-Al-MCM-RT and Mg-Al-MCM-HT did not show the synergistic effect observed by other authors for the aldol condensation reactions over acid-base bifunctional solid catalysts ${ }^{[41-44]}$. There was practically no difference in the activity between Mg-MCM-RT and Mg-Al-MCM-RT and the activity of Mg-Al-MCM-HT was lower than that of Mg-MCM-HT. As discussed above, Mg atoms involved in Mg-MCM-RT are supposed to exist as MgO that is finely dispersed on the surface inside the pores (Scheme 2a), while those in Mg-MCM-HT are supposed to be included in the bulk with the smectite-like structure (Scheme 2b). Because the $\mathrm{Si} / \mathrm{Al}$ ratio was set at a high value of 30 (i.e. the Al loading was low) for Mg-Al-MCM-RT and Mg-Al-MCM-HT, the state of Mg atoms would not be largely affected by the co-incorporation of $\mathrm{Al}$ atoms. $\mathrm{Al}$ atoms incorporated into the silica framework produce acid sites on its surface ${ }^{[13,15,29]}$. Over Mg-Al-MCM-RT, however, those acid sites would be covered by $\mathrm{MgO}$ species that are finely dispersed on the surface, resulting in the absence of the synergistic effect with this catalyst. The octahedral sheet in the structure of smectite, which is supposed to be formed in the bulk of Mg-Al-MCM-HT, can contain not only divalent $\mathrm{Mg}$ cations but also trivalent $\mathrm{Al}$ cations ${ }^{[45]}$. Al atoms co-exiting in the same sheet structure might lower the basic properties of Mg species, causing the lower catalytic activity of Mg-Al-MCM-HT than Mg-MCM-HT.

The preset study has shown that the RT method is excellent for the preparation of superior Mg containing MCM-41 materials. It may also be applicable for the effective incorporation of other hetero atoms into MCM-41. Such study will be reported in future.

\section{Conclusions}

In the present study, one-pot synthesis of Mg containing MCM-41materials was carried out by the RT method and by the HT method, and the differences in their structural and catalytic properties were investigated. Mg-MCM-RT and Mg-Al-MCM-RT have the ordered mesopore structure, large surface areas, and large pore volumes even at a low $\mathrm{Si} / \mathrm{Mg}$ ratio of 
2.7, which correspond to Mg loadings to 20 and $40 \mathrm{wt} \%$ as $\mathrm{MgO}$. At such a low $\mathrm{Si} / \mathrm{Mg}$ ratio of 2.7, Mg-MCM-HT and Mg-Al-MCM-HT have less or no ordered mesopore structure and their surface areas and total pore volumes are much smaller. The Hammet indicator experiments showed that the base strength do not depend on the $\mathrm{Si} / \mathrm{Mg}$ ratio and the basicity of Mg-MCM-RT is stronger than Mg-MCM-HT. These resulted in the higher catalytic activity of Mg-MCM-RT than Mg-MCM-HT as observed for the two model aldol condensation reactions employed. On the basis of the characterization results obtained by XRD, ${ }^{29}$ Si MAS NMR, and UV/Vis spectroscopy, it has been concluded that Mg atoms in Mg-MCM-RT exist as MgO well dispersing on the wall surface of pores, while those in Mg-MCM-HT are included in the bulk with a smectite-like structure. Such a structural difference may result in the distinction of the base strength.

\section{References}

[1] C.T. Kresge, M.E. Leonowicz, W.J. Roth, J.C. Vartuli, J.S. Beck, Nature 359 (1992) 710-713.

[2] C.T. Kresge, J.S. Beck, J.C. Vartuli, W.J. Roth, M.E. Leonowicz, K.D. Schmitt, C.T.W. Chu, D.H. Olson, E.W. Sheppard, S.B. McCullen, J.B. Higgins, J.L. Schlenker, J. Am. Chem. Soc. 114 (1992) 10834-10843.

[3] G.J.A.A. Soler-Illia, C. Sanchez, B. Lebeau, J. Patarin, Chem. Rev. 102 (2002) 4093-4138.

[4] Q.S. Huo, J.L. Feng, F. Schüth, G.D. Stucky, Chem. Mater. 9 (1997) 14-17.

[5] Q. Cai, Z.S. Luo, W.Q. Pang, Y.W. Fan, X.H. Chen, F.Z. Cui, Chem. Mater. 13 (2001) $258-263$.

[6] M. Grün, I. Lauer, K.K. Unger, Adv. Mater. 9 (1997) 254-257.

[7] R. Mokoya, W. Jones, Chem. Commun. (1997) 2185-2186.

[8] C.Y. Chen, H.X. Li, M.E. Davis, Microporous Mater. 2 (1993) 17-26.

[9] R. Schmidt, D. Akporiaye, M. Stöcker, O.H. Ellestad, J. Chem. Soc. Chem. Commun. (1994) 1493-1494.

[10] A. Corma, V. Fornés, M.T. Navarro, J. Pérez-Pariente, J. Catal. 1994, 148, 569-574.

[11] Z.H. Luan, C.F. Cheng, W.Z. Zhou, J. Klinowski, J. Phys. Chem. 99 (1995) 1018-1024.

[12] R. B. Boade, A. Clearfield, Catal. Lett. 32 (1995) 267-272.

[13] Z. Luan, C.F. Cheng, W. Zhou, J. Klinowski, J. Phys. Chem. 99 (1995) 1018-1024.

[14] R. Mokaya, W. Jones, Z. Luan, M.D. Alba, J. Klinowski, Catal. Lett. 37 (1996) 113-120. 
[15] X.S. Zhao, G.Q. Lu, G. J. Millar, Ind. Eng. Chem. Res. 35 (1996) 2075-2090.

[16] F. Shang, H. Liu, J. Sun, B. Liu, C. Wang, J. Guan, Q. Kan, Catal. Commun. 12 (2011) 739-743.

[17] K.M. Reddy, I. Moudrakouski, A. Sayari, J. Chem. Soc. Chem. Commun. (1994) 1059-1060.

[18] P.T. Tanev, M. Chibwe, T.J. Pinnavaia, Nature 368 (1994) 321-323.

[19] A. Corma, M.T. Navarro, J.P. Pariente, J. Chem. Soc. Chem. Commun. (1994) 148-149.

[20] K. Lin, P.P. Pescarmona, H. Vandepitte, D. Liang, G.V. Tendeloo, P.A. Jacobs, J. Catal. 254 (2008) 64-70.

[21] K. Lin, P.P. Pescarmona, K. Houthoofd, D. Liang, G.V. Tendeloo, P.A. Jacobs, J. Catal. 263 (2009) 75-82.

[22] D. Zhao, D. Goldfarb, J. Chem. Soc. Chem. Commun. (1995) 875-876.

[23] L.Z. Wang, J.L. Shi, J. Yu, D.S. Yan, Nanostruct. Mater. 10 (1998) 1289-1299.

[24] Z.Y. Yuan, S.Q. Liu, T.H. Chen, Z.J.Z. Wang, H.X. Li, J. Chem. Soc. Chem. Commun. 9 (1995) 973-974.

[25] A. Sayari, C. Danumah, I.L. Moudrakovski, Chem. Mater. 7 (1995) 813-815.

[26] A. Matsumoto, H. Chen, K. Tsutsumi, M. Grün, K. Unger, Micropor. Mesopor. Mater. 32 (1999) 55-62.

[27] J. Aguado, D.P. Serrano, J.M. Escola, Micropor. Mesopor. Mater. 34 (2000) 45-54.

[28] D.P. Serrano, R.V. Grieken, J. Antonio, A. Galcía, Appl. Catal. A: Gen. 319 (2007) $171-180$.

[29] Z. Wang, Y. Jiang, R. Rachwalik, Z. Liu, J. Shi, M. Hunger, J. Huang, Chem. Chat. Chem. 5 (2013) 3889-3896.

[30] H. Hattori, Chem. Rev. 95 (1995) 537-558.

[31] K. Tanabe, W. Hölderich, Appl. Catal. A: Gen. 181 (1999) 399-434.

[32] H. Hattori, Appl. Catal. A: Gen. 222 (2001) 247-259.

[33] R.J. Davis, J. Catal. 216 (2003) 396-405.

[34] Y. Ono, J. Catal. 216 (2003) 406-415.

[35] J. Weitkamp, M. Hunger, U. Rysma, Micropor. Mesopor. Mater. 48 (2001) 255-270.

[36] A.P. Wight, M.E. Davis, Chem. Rev. 102 (2002) 3589-3614.

[37] F. Hoffmann, M. Cornelius, J. Morell, M. Fröba, Angew. Chem. Int. Ed. 45 (2006) $3216-3251$.

[38] J.I. Yu, S. Yi, A.N. Ko, Catal. Lett. 77 (2001) 165-169.

[39] T. Wang, G. Wu, N. Guan, L. Li, Micropor. Mesopor. Mater. 148 (2012) 184-190. 
[40] J. Tantirungrotechai, P. Thananupappaisal, B. Yoosuk, N. Viriya-empikul, K. Faungnawakij, Catal. Commun. 16 (2011) 25-29.

[41] R.K. Zeidan, S.J. Hwang, M.E. Davis, Angew. Chem. Int. Ed. 45 (2006) 6332-6335.

[42] R.K. Zeidan, M.E. Davis, J. Catal. 247 (2007) 379-382.

[43] E.L. Margelefsky, R.K. Zeidan, M.E. Davis, Chem. Soc. Rev. 37 (2008) 1118-1126.

[44] X. Yu, Y. Zou, S. Wu, H. Liu, J. Guan, Q. Kan, Mater. Res. Bul. 46 (2011) 951-957.

[45] S. Fujita, B.M. Bhanage, D. Aoki, Y. Ochiai, N. Iwasa, M. Arai, Appl. Catal. A: Gen. 313 (2006) 151-159.

[46] S. Kvisle, A. Aguero, R.P.A. Sneeden, Appl. Catal. 43 (1988) 117-131.

[47] W. Janssens, M. Ekaterina, V. Pieter, D.C. Filip, H. Kristof, K. Stef, J.A. Martens, J.A. Pierre, B.F. Sels, Chem. Sus. Chem. 8 (2015) 994-1008.

[48] C. Angelici, M.E.Z. Vethoen, B.M. Weckhuysen, P.C.A. Bruijnincx, Chem. Sus. Chem. 7 (2014) 2505-2515.

[49] S. Colluccia, A.J. Tench, R.L. Segall, J. Chem. Soc. Faraday Trans. 175 (1979) 1769-1779.

[50] G.E. Maciel, D.W. Sindrof, J. Am. Chem. Soc. 102 (1980) 7606-7607.

[51] K.J.D. MacKenzie, R.H. Meinhold, Am. Miner. 79 (1994) 43-50.

[52] K.J.D. MacKenzie, S. Bradley, J.V. Hanna, M.E. Smith, J. Mater. Sci. 48 (2013) 1787-1793.

[53] L. Chen, G. Yen, W. Zhou, J. Dijkmans, B. Sels, A. Malfliet, M. Guo, Ceram. Int. 41 (2015) 12651-12657.

[54] S. Fujita, M. Tanaka, M. Arai, Catal. Sci. Technol. 4 (2014) 1563-1569. 


\section{Figure and table captions}

Table 1 Textural properties of MCM-41 materials prepared

Scheme 1. (a) Aldol condensation of nitrobenzaldehyde and acetone and (b) self-condensation of acetone.

Scheme 2. States of Mg atoms in (a) Mg-MCM-RT and (b) Mg-MCM-HT.

Fig. 1. Pore size distribution curves of some selected MCM-41 samples prepared by the RT method and by the HT method. The Si/Mg ratio was 2.7 for all $\mathrm{Mg}$ containing samples and the $\mathrm{Si} / \mathrm{Al}$ ratio was 30 for all $\mathrm{Al}$ containing samples.

Fig. 2. Small-angle XRD patterns of some selected MCM-41 samples prepared (a) by the RT method and (b) by the HT method. The Si/Mg ratio was 2.7 for all Mg containing samples and the $\mathrm{Si} / \mathrm{Al}$ ratio was 30 for all $\mathrm{Al}$ containing samples. The patterns were moved vertically for clarity.

Fig. 3. TEM images of Mg-Al-MCM-RT and Mg-Al-MCM-HT. For both the samples, the $\mathrm{Si} / \mathrm{Mg}$ and $\mathrm{Si} / \mathrm{Al}$ ratios were 2.7 and 30 , respectively.

Fig. 4. Effects of the Mg loading and the preparation method on the catalytic activity of $\mathrm{Mg}$ incorporated MCM-41 for the aldol condensation of 4-nitrobenzaldehyde and acetone. (०) Mg-MCM-RT, (•) Mg-Al-MCM-RT, (口) Mg-MCM-HT, (•) Mg-Al-MCM-HT. Reaction conditions: 4-nitrobenzaldehyde $0.5 \mathrm{mmol}$, acetone $10 \mathrm{~mL}$, catalyst $50 \mathrm{mg}, 353 \mathrm{~K}, 7 \mathrm{~h}$.

Fig. 5. Comparison between (०) Mg-MCM-RT and ( $\square$ ) Mg-MCM-HT in the catalytic activity for the self-condensation of acetone. Reaction conditions: acetone $10 \mathrm{~mL}$, catalyst $50 \mathrm{mg}, 353$ K, 2 h.

Fig. 6. Large-angle XRD patterns of Mg-MCM-RT and Mg-MCM-HT. Numbers in the figure represent the $\mathrm{Si} / \mathrm{Mg}$ ratios in the samples examined. The patterns were moved vertically for clarity.

Fig. 7. Diffuse reflectance UV-vis spectra of (a) Mg-MCM-RT and (b) Mg-MCM-HT. Si/Mg ratio was 2.7 for both samples.

Fig. 8. ${ }^{29}$ Si MAS NMR spectra of (a) Mg-MCM-RT and (b) Mg-MCM-HT. Si/Mg ratio was 2.7 for both samples.

Fig. 9. ${ }^{29}$ Si CP MAS NMR spectra (a) of Mg-MCM-RT and (b) Mg-MCM-HT. Si/Mg ratio was 2.7 for both samples. 
Table list:

Table 1 Textural properties of MCM-41 materials prepared

\begin{tabular}{|c|c|c|}
\hline Sample & $\begin{array}{c}S_{\mathrm{BET}} \\
\left(\mathrm{m}^{2} / \mathrm{g}\right)\end{array}$ & $\begin{array}{c}V_{\text {pore }} \\
\left(\mathrm{cm}^{3} / \mathrm{g}\right)\end{array}$ \\
\hline MCM-RT & 1045 & 1.52 \\
\hline Mg-MCM-RT & 883 & 1.22 \\
\hline Mg-Al-MCM-RT ${ }^{\mathrm{a}, \mathrm{b}}$ & 852 & 1.53 \\
\hline MCM-HT & 872 & 0.87 \\
\hline Mg-MCM-HT & 556 & 0.92 \\
\hline Mg-Al-MCM-HT ${ }^{\mathrm{a}, \mathrm{b}}$ & 483 & 1.02 \\
\hline
\end{tabular}


(a)

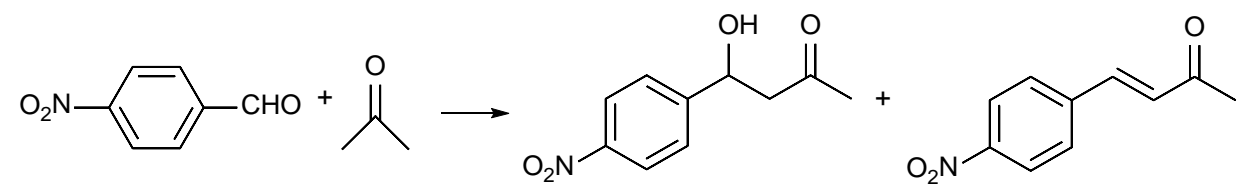

(b)<smiles>CC(=O)C=C(C)C</smiles>

Scheme 1 (a) Aldol condensation of nitrobenzaldehyde and acetone and (b) self-condensation of acetone.

(a)

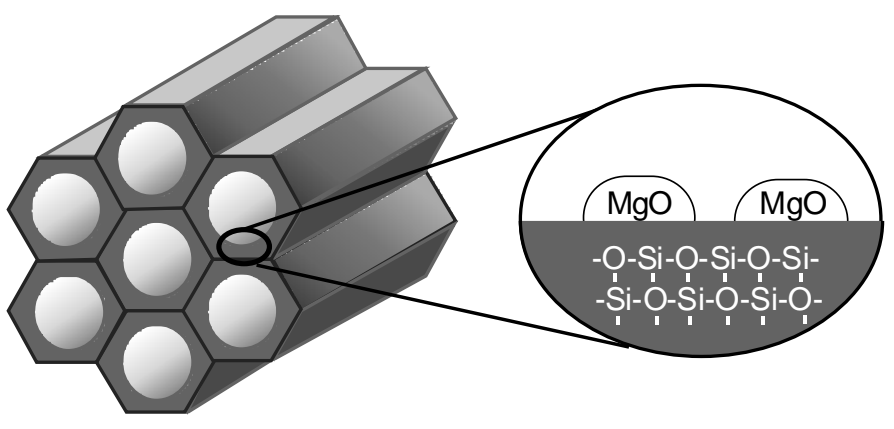

(b)

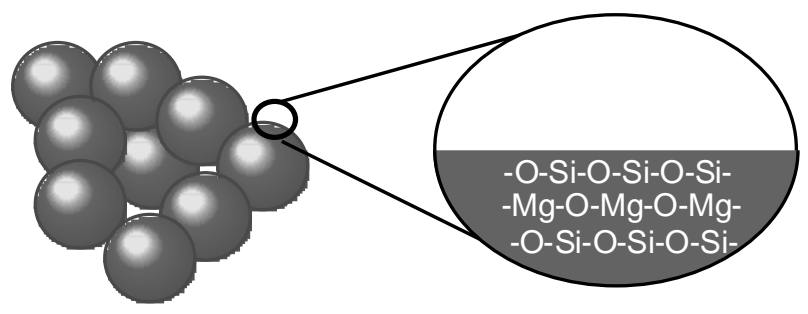

Scheme 2. States of Mg atoms in (a) Mg-MCM-RT and (b) Mg-MCM-HT. 


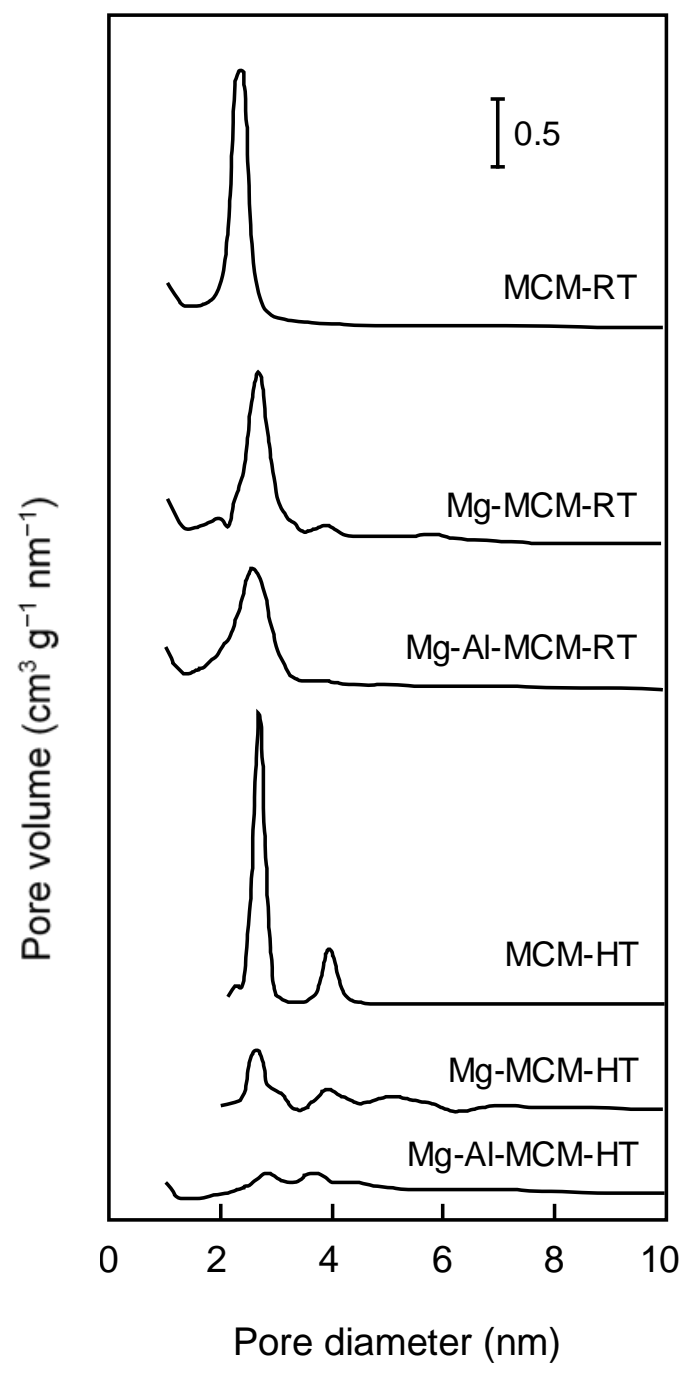

Fig. 1. 

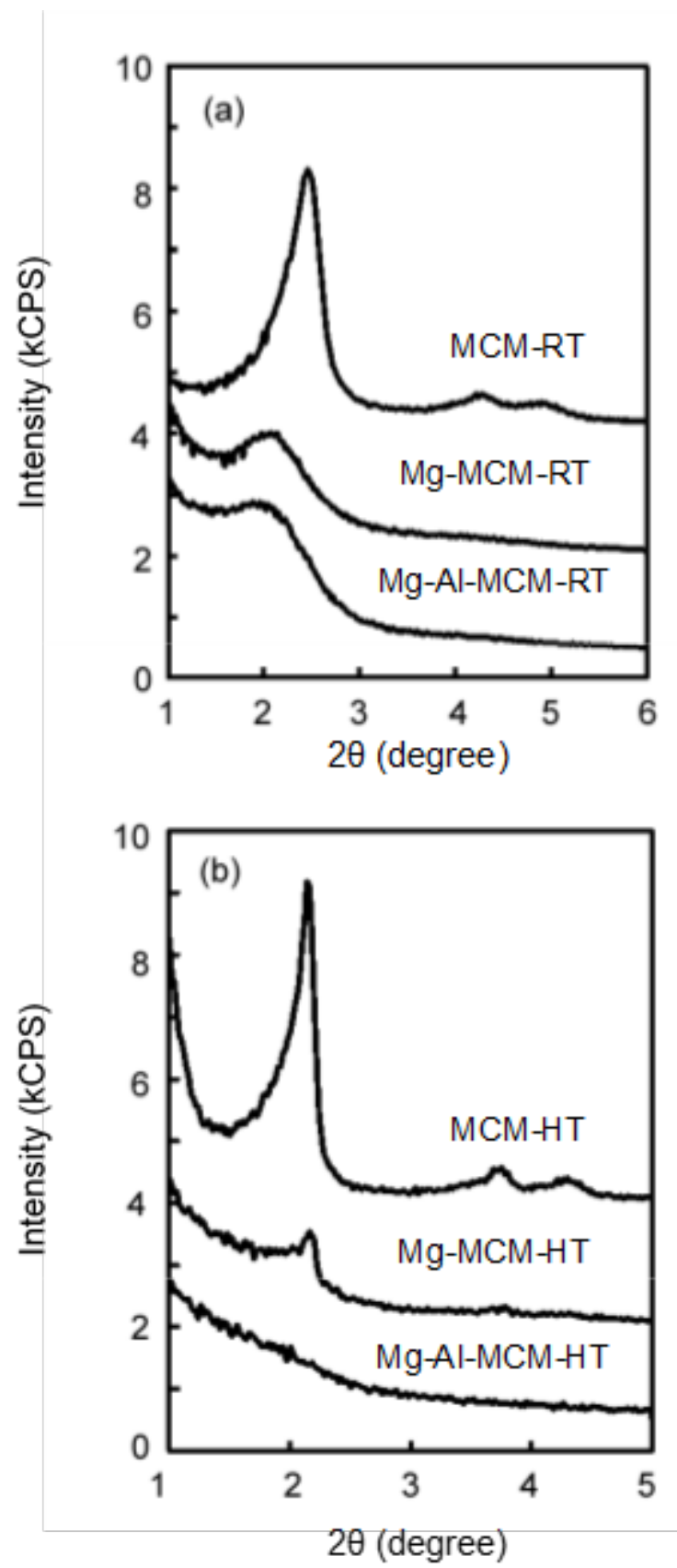

Fig. 2. 
(a) Mg-Al-MCM-RT

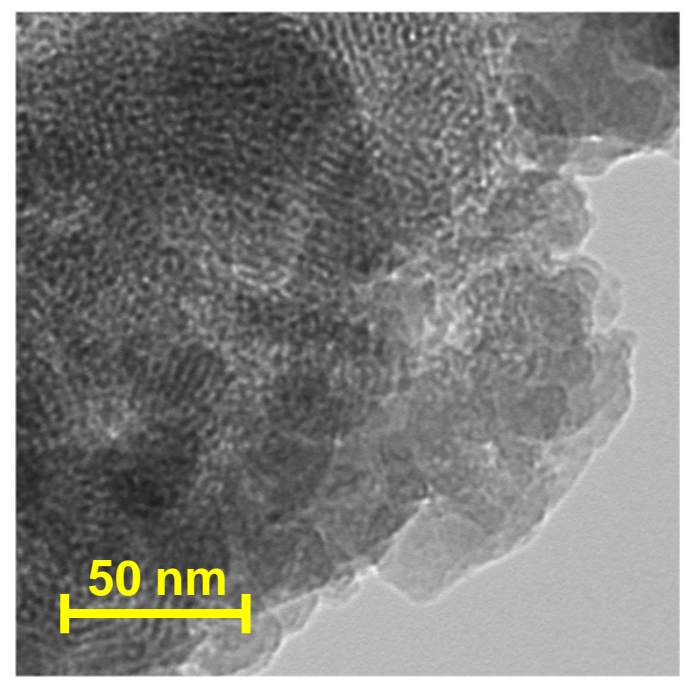

(b) Mg-Al-MCM-HT

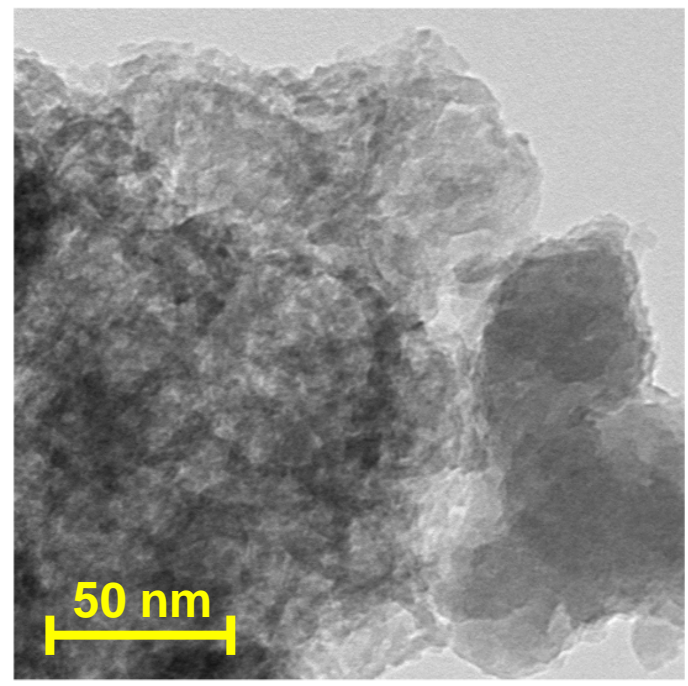

Fig. 3. 


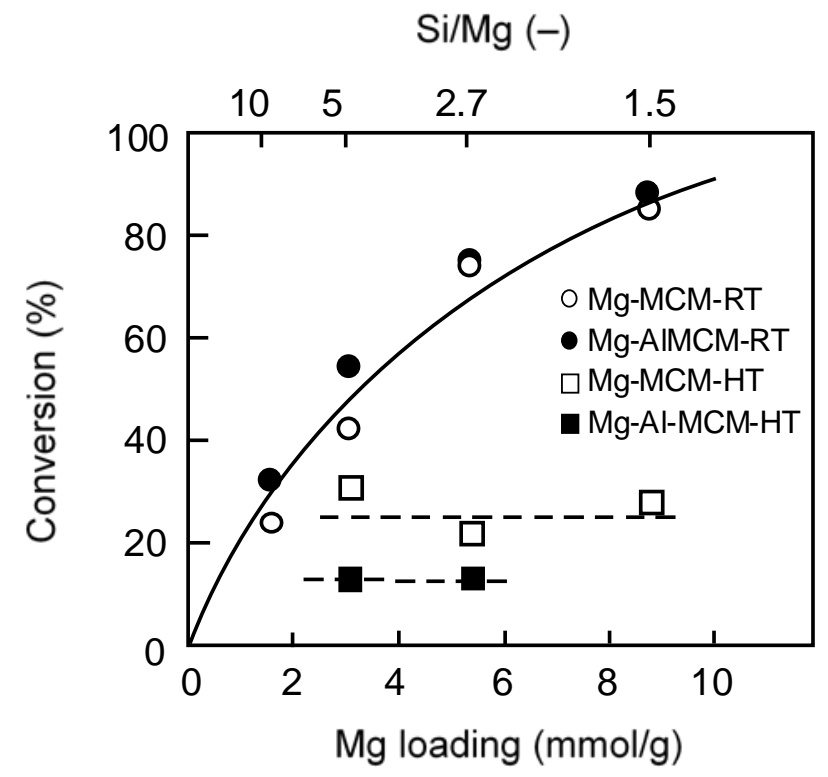

Fig. 4.

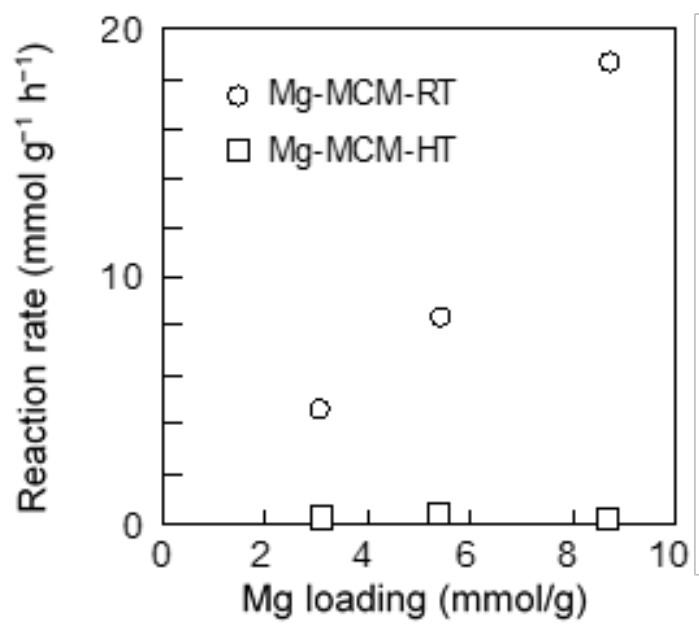

Fig. 5. 


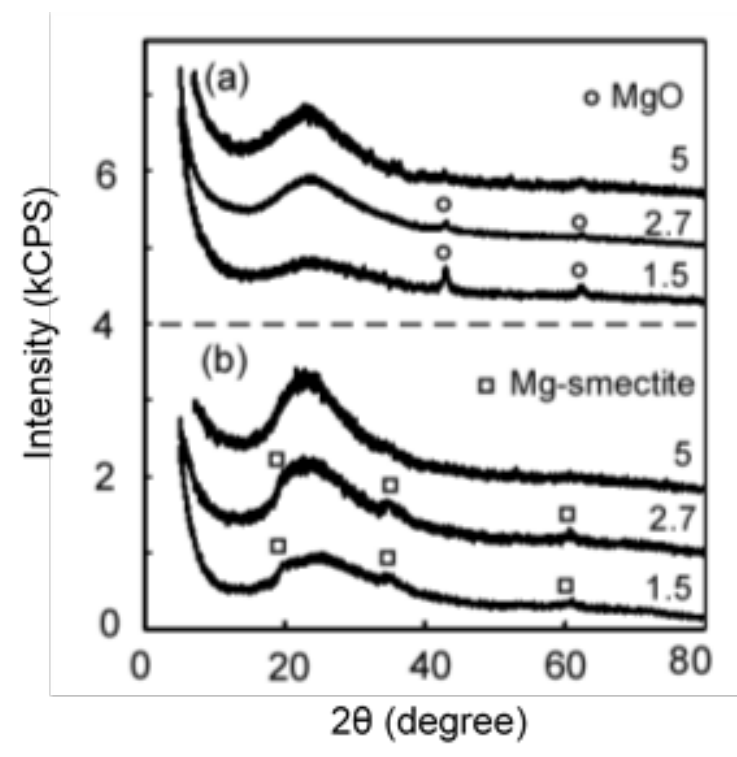

Fig. 6.

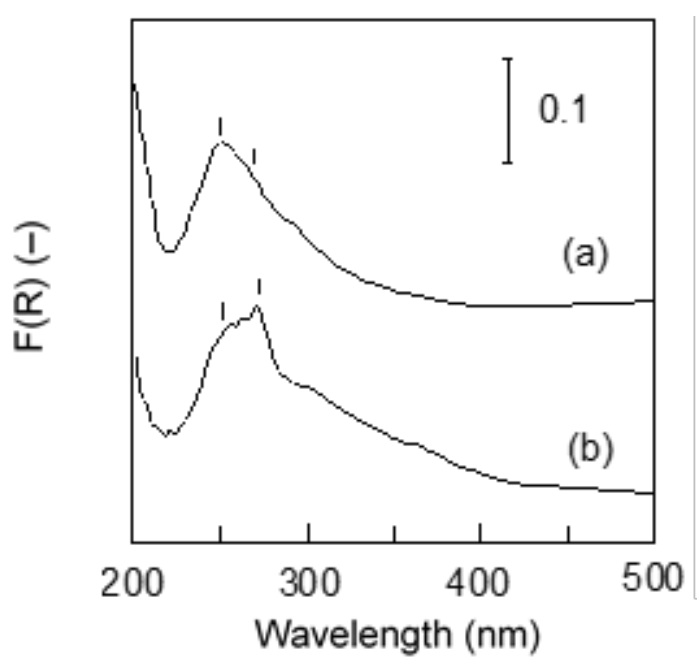

Fig. 7. 

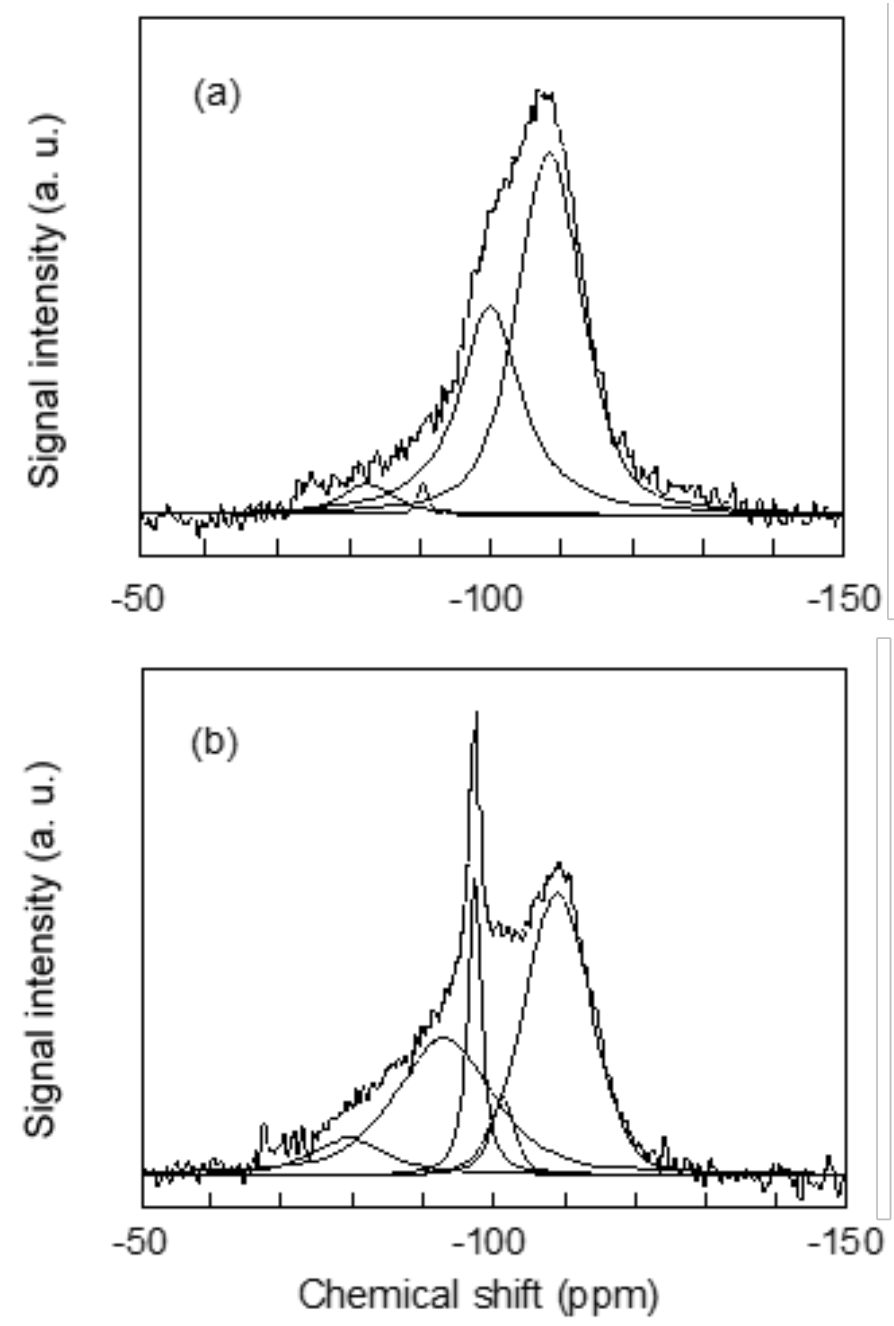

Fig. 8.

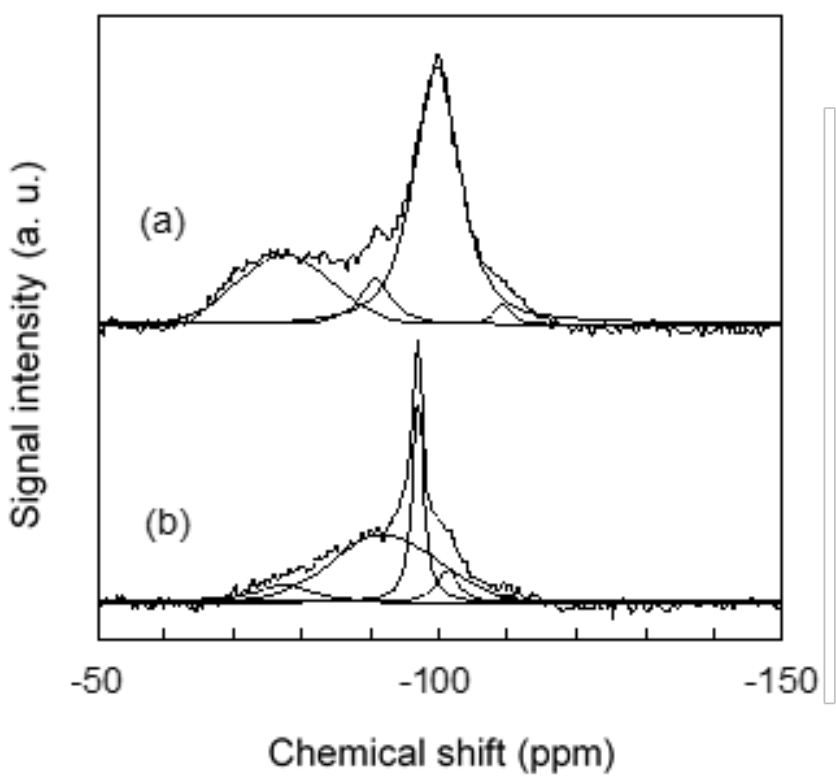

Fig. 9. 